\title{
Video-rate capture of Dynamic Face Shape and Appearance
}

\author{
Ioannis A. Ypsilos \\ Centre for Vision Speech and Signal Processing \\ University of Surrey, Guildford, GU2 7XH, UK
}

\author{
Simon Rowe \\ Canon Research Centre Europe \\ Bracknell, Beskshire \\ RG12 2XH, UK
}

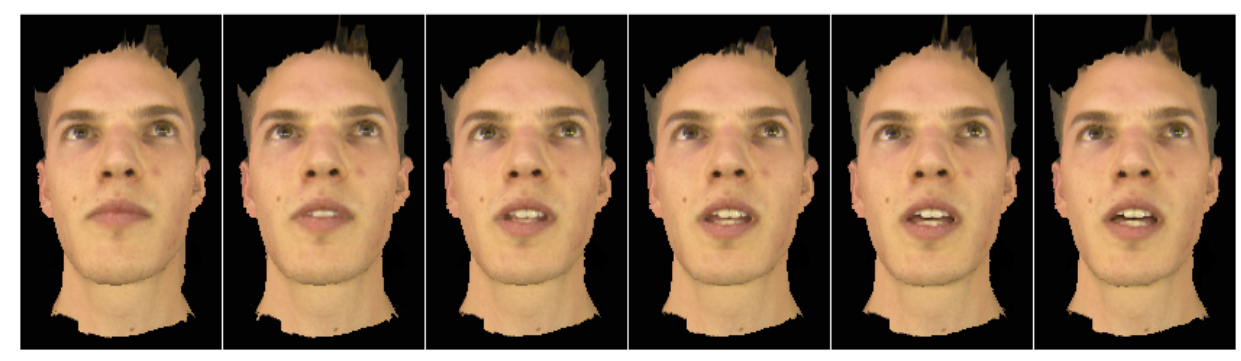

\begin{abstract}
This paper presents a system for simultaneous capture of video sequences of face shape and colour appearance. Shape capture uses a projected infra-red structured light pattern together with stereo reconstruction to simultaneously acquire full resolution shape and colour image sequences at video rate. Displacement mapping techniques are introduced to represent dynamic face surface shape as a displacement video. This unifies the representation of face shape and colour. The displacement video representation enables efficient registration, integration and spatiotemporal analysis of captured face data. Results demonstrate that the system achieves video-rate $(25 \mathrm{~Hz})$ acquisition of dynamic $3 D$ colour faces at PAL resolution with an rms accuracy of $0.2 \mathrm{~mm}$ and a visual quality comparable to the captured video.
\end{abstract}

\section{Introduction}

The acquisition of realistic and accurate animated face models has received considerable interest over the past decade. Potential applications include entertainment, communication, medical analysis and facial biometrics. Producing convincing 3D animated models of facial dynamics for speech and expressions is a difficult problem due to the sensitivity of human perception to the nuances of face appearance and movement. In the entertainment industry highly realistic animated face models have been achieved for film production but require months of skilled manual animation to obtain believable results. Research in computer vision has addressed the problem of reconstructing face models of real people from images using both active and passive sensing.
Current approaches enable either reconstruction of a realistic face model for a single rigid pose or reconstruction of the movement of sparse facial features. To date there is no solution which allows simultaneous capture of the detailed shape deformation and colour appearance of a real face during speech or expression. In this paper we introduce a novel capture system which achieves video-quality $3 \mathrm{D}$ reconstruction of real faces as shown above. This is the first system to demonstrate simultaneous video-rate capture of shape and appearance. The captured models provide a starting point for modelling and analysis of facial surface dynamics.

Recent research has resulted in systems for video-rate dense 3D shape acquisition using both active $[10,14,15,9]$ and passive $[7,11,17]$ techniques. Due to the uniform appearance of large regions of the face, passive techniques such as multiple view stereo [7] can not reconstruct accurate shape. Active sensors produce relatively accurate high-resolution surfaces measurements but require the projection of a visible pattern onto the object surface prohibiting simultaneous capture of surface colour appearance. The sensor technology introduced in this paper uses infra-red structured light to allow simultaneous acquisition of highresolution shape and colour at video frame rates.

Passive face reconstruction techniques have been developed to reconstruct animated models of the face from images [2] and video [3, 16]. Blanz and Vetter [2] used learnt statistical models of 3D face shape and appearance to reconstruct photo-realistic 3D face models from a single image. Other face reconstruction $[3,16]$ use model-based bundle adjustment techniques to reconstruct realistic static face models from image sequences. Currently these approaches 
are limited to reconstruction of static face shape for a single pose.

Pighin et al.[13] used marker based capture to accurately reconstruct the movement of a sparse set of 3D points on the face surface. The marker movement was then used to morph a face model reproducing the coarse facial dynamics. To overcome the limitation of sparse reconstruction Kalbera and Van Gool [6] combine markers with active structured light capture to acquire dense facial shape deformation for animation. Due to the use of visible markers and structured light previous approaches do not allow simultaneous capture of facial appearance. The shape capture system introduced in this paper uses infra red structured light to allow simultaneous capture of shape and appearance. Automatic facial alignment is used to avoid the requirement for known marker correspondence.

\section{Video-rate Capture}

In this section we present the active sensor technology developed to allow simultaneous video-rate capture of shape and colour. Infra red (IR) structured light illumination together with IR stereo acquisition is used to capture shape. Colour is captured simultaneously using the visible spectrum with uniform white-light illumination.

\subsection{Capture System}

Figure 1 shows a schematic of the capture system. The system comprises three units each capturing shape and colour at video-rate designed to give ear-to-ear face coverage. Each unit comprises a colour camera, IR projector and two IR cameras.

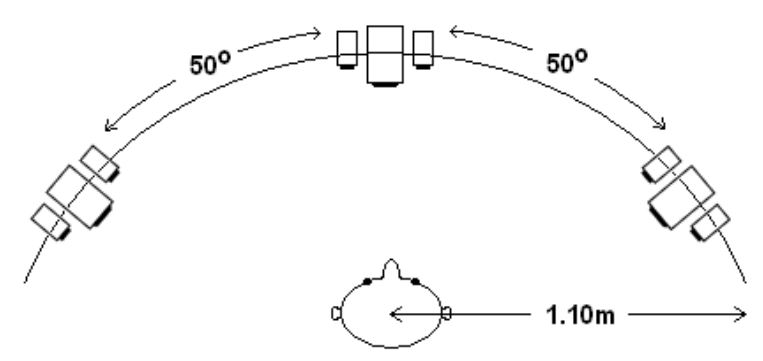

Figure 1: Schematic of the capture equipment

Shape is captured using active projection of a random dot IR structured light pattern onto to the face together with IR stereo capture. Stereo correspondence is then used to reconstruct the surface shape providing a single-shot shape capture technology. IR light is projected using a conventional slide projector (NOBO SPII) fitted with an IR-pass filter (Hoya R72) which eliminates projection of visible light. Pattern projection is achieved by fitting each projector with a $35 \mathrm{~mm}$ slide containing a random dot pattern. The projected pattern is invisible to the human eye. IR stereo image capture uses Sony XC8500 cameras fitted with the same IR-pass filters (Hoya R72) giving progressive scan PAL resolution $(768 \times 576)$ at frame rates up to $50 \mathrm{~Hz}$. Figure 2 shows overlap of the CCD response and IR filter transmittance characteristics.

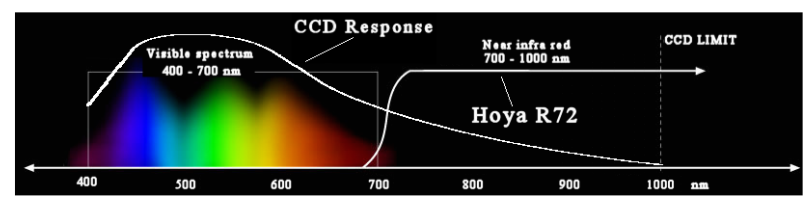

Figure 2: Spectral distribution of the infra red filters and Sony XC8500 CCD sensitivity

Colour capture uses Sony DXC9100P 3CCD cameras to acquire progressive scan PAL resolution video at frame rates up to $50 \mathrm{~Hz}$. The colour cameras have a built in IR-cut filter which prevents the projected IR illumination distorting the colour images. Uniform white-light illumination of the face is achieved using 'cold' fluorescent lights, this avoids the projection of additional IR illumination onto the face which would saturate the projected pattern.

All cameras are synchronised via a common genlock synchronisation pulse. Captured video frames are also timecoded to check for dropped frames. Currently the system operates at full PAL resolution $25 \mathrm{~Hz}$ progressive scan using standard PAL video capture cards. Figure 3 shows a typical set of captured images, 3 IR stereo pairs and 3 colour, of a persons face. Calibration of all cameras is performed using public camera calibration software, Zhang [18], to estimate intrinsic and extrinsic parameters. Calibration gives an rms re-projection error of less than 0.5 pixels across the capture volume.

\subsection{Stereo Surface Reconstruction}

For each pair of IR cameras we estimate stereo correspondence and reconstruct the visible surface shape in the following steps:

1. Rectification: Images are rectified to align the epipolar lines horizontally between views.

2. Coarse correlation: Coarse window matching along epi-polar lines using a $13 \times 13$ window at every $n^{t h}$ pixel $(n=5)$

3. Dense adaptive correlation: Adaptive window matching along the epi-polar line to identify all correlation peaks above a threshold value $(0.75)$. Window sizes are varied between $5 \times 5$ in regions of highgradient $g \geq 0.8$ upto $13 \times 13$ in regions of low gradi- 
ent $g \leq 0.2$ with corresponding intermediate window sizes of 7,9 and 11 .

4. Neighbourhood consistency: All pixels with a single correlation peak are accepted, pixels with multiple correlation peaks are resolved based on consistency with adjacent pixels for a smooth surface.

5. Bi-direction consistency: Only pixels with the same correspondence matching from left to right image and right to left image are accepted [4].

6. Sub-pixels disparity estimation: A quadratic function is fitted to the correspondence estimates over a 5 pixel neighbourhood.

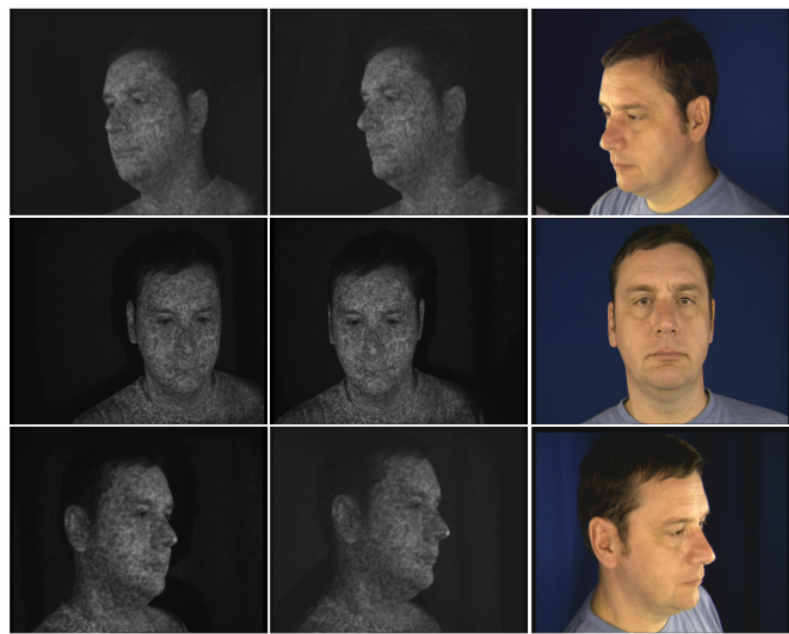

Figure 3: Captured IR stereo pairs and colour images

The stereo correspondence algorithm is based on the adaptive window technique [12] that changes the window size according to the local disparity gradient. As the IR images have a dense texture pattern we use a two pass approach which gives improved computational efficiency and reliable correspondence. Initially we use a coarse pass of the image with a relatively large window size to estimate correspondence at every $n^{\text {th }}$ pixel. Normalised crosscorrelation is used to measure image similarity. Coarse correspondence is used to estimate the local surface gradient, $g$, with-respect to the stereo views, where $0 \leq g \leq 1$. A second detailed pass is then performed at every pixel adapting the correlation window size according to the local surface gradient, $g$. The use of adaptive window sizes gives accurate correspondence according to local surface geometry minimising the effects of errors due to noise and surface orientation.

Neighbourhood consistency is used to resolve ambiguities where multiple correlation peaks are found. Initially pixels with only one peak above the correlation threshold

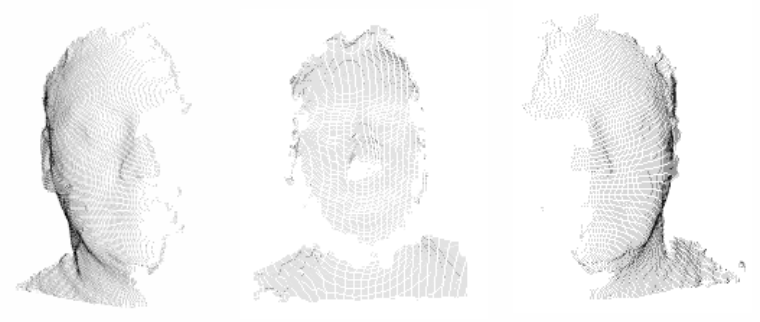

Figure 4: 3D surface points reconstructed from stereo. (28002 points in total.)

are accepted. Correspondence for adjacent pixels with multiple peaks are then resolved to be consistent with a smooth surface. Stereo correspondence between each pair of IR cameras results in three sets of surface measurements captured at video-rate as illustrated in Figure 4.

\section{Dynamic Surface Representation}

The capture system acquires multiple sets of facial surface measurements with corresponding colour images at videorate. In this section we address the problem of integration of surface measurements into a single representation and temporal registration of face sequences.

\subsection{Displacement Mapping}

Displacement mapping represents the detailed surface shape as displacements between a simple surface primitive (plane, sphere, cylinder) and the detailed surface. The assumption is that a unique one-to-one (bi-jective) mapping exists between the detailed and primitive surfaces. In this work an ellipsoidal surface primitive is used which provides a simple mapping between the captured data and a 2D 'displacement image' domain. The ability to accurately represent dynamic face shape is verified in section 4 .

An ellipsoid is defined by the location of its centre, $\vec{c}$ together with the magnitude and direction of the three orthogonal axis vectors, $\vec{v}_{1}, \vec{v}_{2}, \vec{v}_{3}$ giving 9 degrees-of-freedom (6 pose and 3 radii). At each frame we capture a set of face surface measurement points $X(t)=\left\{\vec{x}_{i}^{t}\right\}_{i=1}^{N_{t}}$ where $\vec{x}=(x, y, z) \in \Re^{3}$. For the first frame $t=0$ we assume that the face is roughly aligned with the world coordinate system so that the frontal direction is looking towards the positive z-axis. The ellipsoid's centre is then defined as $\left.\vec{c}^{0}=\left(\operatorname{Mean}\left\{x_{i}^{0}\right\}_{i=1}^{N_{0}}, \operatorname{Mean}_{\{} y_{i}^{0}\right\}_{i=1}^{N_{0}}, \operatorname{Min}\left\{z_{i}^{0}\right\}_{i=1}^{N_{0}}\right)$ and the three axis are aligned to the world coordinate system with lengths $\left|\vec{v}_{1}^{0}\right|=2 \times\left|\vec{v}_{2}^{0}\right|=2 \times\left|\vec{v}_{3}^{0}\right|$. Temporal registration, presented in section 3.3, is used to define the ellipsoid centre and axes in subsequent frames, $\left(\vec{c}^{t},\left\{\vec{v}_{i}^{t}\right\}_{i=1}^{3}\right)$, from the estimated face pose (rotation, translation). 
A sampled displacement map image representation, $D_{t}(i, j)$ for $i=1 \ldots N_{i}, j=1 \ldots N_{j}$, of the surface shape for each frame is then computed by sampling the distance to captured data along equiangular rays from the ellipsoid centre: $\vec{v}(i, j)=$ $\left(\cos \left(\phi_{j}\right) \cos \left(\theta_{i}\right), \cos \left(\phi_{j}\right) \sin \left(\theta_{i}\right), \sin \left(\phi_{j}\right)\right)$ where $\theta_{i}=$ $\left(\frac{2 i\left(\pi-\theta_{\min }\right)}{N_{i}}+\theta_{m i n}\right), \phi_{j}=\left(\frac{2 j\left(\pi-\phi_{m i n}\right)}{N_{j}}+\phi_{m i n}\right)$, and $\theta_{\min }=\phi_{\min }=\frac{\pi}{2}$. The rays $\vec{v}(i, j)$ provide a non-uniform sample of the ellipsoid surface such that there is greater sampling in the centre of the face as illustrated in Figure 5 . The measured surface is represented by a displacement image $D_{t}(i, j)$ by sampling the distance from the ellipsoid centre $\vec{c}_{t}$ to the measured data along each ray $v(i, j)$ such that the intersection $\vec{x}$ with the measured surface is given by $\vec{x}=\vec{c}_{t}+D_{t}(i, j) v(i, j)$.

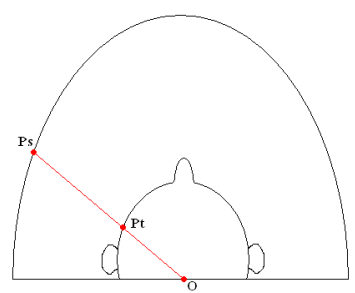

Figure 5: Mapping the geometry of a face to an ellipsoid

To intersect the ray $v(i, j)$ with the surface we first compute a discontinuity constrained triangulation [5]. This triangulates the measured surface points for each stereo pair $\left\{\vec{X}^{p}(t)\right\}_{p=1}^{3}$ into a triangulated mesh $M_{t}^{p}$ based on their adjacency in the stereo depth map. Adjacent points are connected only if the distance between them is less than three times the spatial sampling resolution (minimum distance between adjacent points). This provides a conservative triangulation which approximates the local surface continuity but does not triangulate across depth discontinuities. For each mesh $M_{t}^{p}$ the displacement image $D_{t}^{p}(i, j)$ is constructed sampling the distance along the rays $\vec{v}(i, j)$.

\subsection{Integration of Face Shape and Colour}

Two problems must be addressed to obtain a single model for each time frame: integration of multiple view surface measurements $M_{t}^{p}$ from each stereo pair; and integration of colour images into a single texture map. Having constructed a displacement map representation $D_{t}^{p}(i, j)$ for each stereo pair the problem is to combine them into a single surface representation. The displacement image representation provides a simple mechanism for integration of overlapping surface measurements by combining their corresponding displacement values:

$$
D_{t}(i, j)=\sum_{p=1}^{3} w_{t}^{p}(i, j) D_{t}^{p}(i, j)
$$

where $w_{t}^{p}(i, j)=0$ if no surface measurement is obtained along $\vec{v}_{t}(i, j)$ for the $p^{t h}$ stereo pair, otherwise $w_{t}^{p}(i, j)$ is proportional to the confidence in the surface measurement and $\sum w_{t}^{p}(i, j)=1$. This provides a computationally efficient method for fusion of the face surface measurements into a single face surface representation $D_{t}(i, j)$. The integrated face displacement map can be rendered as a single triangulated mesh $M_{t}$ by triangulating adjacent pixels in the displacement image $D_{t}(i, j)$ and re-projecting along the corresponding rays $v(i, j)$ to obtain the vertex positions: $\vec{x}_{i j}=\vec{c}_{t}+D_{t}(i, j) v(i, j)$.

The elliptical mapping also provides an efficient mechanism for integration of the three colour images into a single texture map. The registration of the three colour images with the reconstructed surface shape is known from the colour calibration giving overlapping texture maps from each image $I^{p}(i, j)$. The problem is then to integrated the texture maps in the overlapping regions. Initially we correct for differences in camera colour response by fitting a linear model of the gain and offset between overlapping regions of the texture images relative to the central camera: $r_{t}^{C}(i, j)=\vec{a}_{t} \vec{r}_{t}^{O}(i, j)+\vec{b}_{t}$ where $\vec{r}_{t}^{C}(i, j)$ and $\vec{r}_{t}^{0}(i, j)$ are the rgb colour response of the centre and overlapping cameras. The gain and offset parameters are $\left[\vec{a}_{t}, \vec{b}_{t}\right]$ are estimated using least-squares from the difference in colour response of all overlapping pixels. Given accurate registration and correction for colour response a simple blending colour [8] of overlapping pixels is then performed.

$$
I_{t}(i, j)=w^{C}(i, j) r_{t}^{C}(i, j)+w^{O}(i, j)\left(\vec{a}_{t} r_{t}^{O}(i, j)+\vec{b}_{t}\right)
$$

where $w_{t}^{C}(i, j)$ and $w_{t}^{O}(i, j)$ are weights for the centre and overlap colour images respectively. Weights are set such that in the central and side face region only the centre or side images are used giving the highest sampling resolution. Blending then occurs in two bands $\left(\pi-\theta_{\text {blend }}-\theta_{\text {width }}\right) \leq$ $\theta \leq\left(\pi-\theta_{\text {blend }}\right)$ and $\left(\pi+\theta_{\text {blend }}\right) \geq \theta \geq \pi\left(\pi+\theta_{\text {blend }}+\right.$ $\left.\theta_{\text {width }}\right)$ with $\theta_{\text {blend }}=\frac{\pi}{8}$ and $\theta_{\text {width }}=\frac{\pi * 9}{N_{i}}$ giving a 9 pixel blending band on either side. A half cycle of a sine function over the blending band is used to give a smooth transition: $w^{C}(i, j)=\frac{1}{2}\left(1+\sin \left(\frac{\left(\theta_{i}+\theta_{b l e n d}+\theta_{\text {width }}\right) \pi}{2 * \theta_{\text {width }}}\right)\right.$ and $w^{O}(i, j)=\left(1-w^{C}(i, j)\right)$. Figure 6 shows results of the shape and colour integration to reconstruct a single $3 \mathrm{D}$ colour face model at each time.

\subsection{Temporal Registration}

In this section we introduce an efficient method for temporal registration of surface shape to estimate the head pose. 


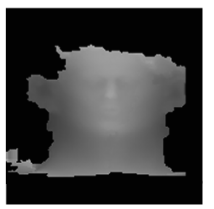

(a)

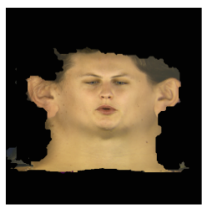

(b)

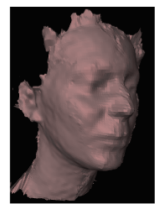

(c)

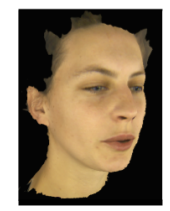

(d)
Figure 6: Combining texture and shape to develop the complete 3D face. (a) Displacement map, (b) Texture map, (c) 3D shape, (d) Textured 3D face.

Temporal registration is used to transform the ellipsoid parameters at each time to obtain an aligned displacement map representation for temporal analysis of shape deformation. Variations of the Iterative Closest Point (ICP) algorithm $[1,15]$ are widely used for surface shape registration to estimate the rigid transformation $T_{t q}$ between two surfaces $S_{p}$ and $S_{q}$. ICP iteratively estimates the transformation $T_{p q}$ by minimising the sum of nearest point distance between the surface $S_{t}$ and the transformed surface $T_{t q}\left(S_{q}\right)$. The computational cost is primarily dependant on the cost of evaluating closest point computations between overlapping surfaces in $\Re^{3}$. Volumetric partitioning schemes such as octrees have previously been used to reduce the complexity to $O\left(N^{2} \log N\right)$ for $N$ point searches.

In this work we use the displacement map representation to compute approximate closest points for overlapping surface regions in constant time. The distance $d(i, j)$ between two surfaces $D_{p}(i, j)$ and $D_{q}(i, j)$ at point $(i, j)$ for an estimated transformation $\hat{T}_{p q}$ is taken as the difference of their displacement values: $d(i, j)=D_{p}(i, j)-\hat{T}_{p q}\left(D_{q}(i, j)\right)$ which requires a simple lookup of the corresponding displacement image pixels. This reduces the computational complexity of registration to be linear $O(N)$ in the number of overlapping points $N$.

ICP using the displacement map distance is used to recursively estimate the change in head pose between successive frames $T_{t-1, t}$. An estimate of the transformation between the initial pose and the $t^{t h}$ frame can then be obtained as the product of intermediate transformations $\tilde{T}_{0, t}=\prod_{s=1}^{t} T_{s-1, s}$. The initial estimate is refined by direct ICP registration between distance functions $D_{0}(i, j)$ and $T_{0, t}\left(D_{t}(i, j)\right)$ to eliminate propagation of errors. The estimated pose $T_{0, t}$ is used to define the ellipsoid parameters at time $t$ as $\left(\vec{c}^{t}=T_{0, t} \vec{c}^{0},\left\{\vec{v}_{i}^{t}=T_{0, t} \vec{v}_{i}^{0}\right\}_{i=1}^{3}\right)$, giving a registered displacement image $D_{t}(i, j)$. This registration forms the basis for subsequent temporal analysis of nonrigid surface shape change.

\subsection{Temporal Filtering}

The displacement map representation together with temporal registration provides an image sequence of surface shape and appearance over time. This representation can be used to analyse the spatio-temporal characteristics of face shape. Initial work has addressed the problem of noise removal from the captured shape data. In this work we use temporal averaging to improve estimates for non-rigid surfaces by removing noise. As the surfaces are registered we ensure that corresponding points are averaged over time. A spatio-temporal window of $n \times m \times T$ where $n \times m$ and $T$ are spatial and temporal resolution respectively. Spatiotemporal smoothing is performed by convolution with a filter $f()$ :

$$
D_{t}^{S}(i, j)=\sum_{r=i-\frac{n}{2}}^{i+\frac{n}{2}} \sum_{s=j-\frac{m}{2}}^{j+\frac{m}{2}} \sum_{\tau=t-\frac{T}{2}}^{t+\frac{T}{2}} D_{t}(r, s) f(r, s, \tau)
$$

where $f(r, s, \tau)$ is a Gaussian smoothing kernel. Figure 7 shows the shape reconstruction for frames from a sequence with and without temporal smoothing. Results show that temporal smoothing reduces measurement noise in individual frames without significant loss of spatial resolution.

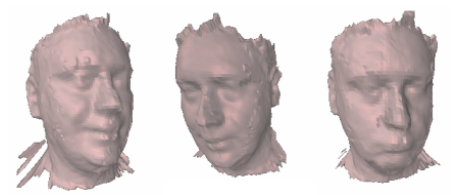

(a) before filtering

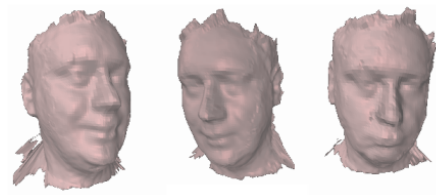

(b) after filtering

Figure 7: Temporal filtering with a window of size 5.

\section{Results}

The dynamic face capture system has been used to capture a database of 51 people performing facial expressions and speech. Figures 8 and 9 show typical sequences reconstructed for two people ${ }^{1}$. Results demonstrate that the ellipsoidal displacement map representation together with temporal registration successfully integrates and registers the non-rigid face shape for a wide variety of facial dynamics captured. Shape reconstruction gives an rms error of approximately $0.2 \mathrm{~mm}$ for surface measurement error estimated between successive frames. Reconstruction time for integration, temporal registration and filtering is less than 1 second per frame on a PentiumIII 900MHz machine.

\footnotetext{
${ }^{1}$ Please see video of results submitted.
} 

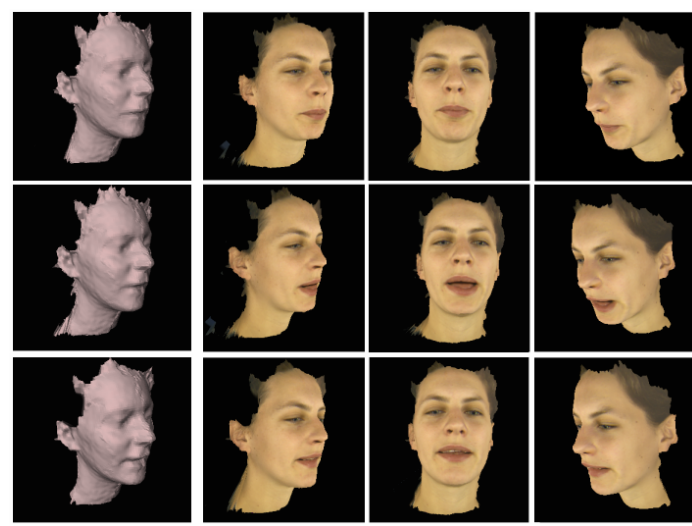

Figure 8: 3D Reconstructions of facial speech

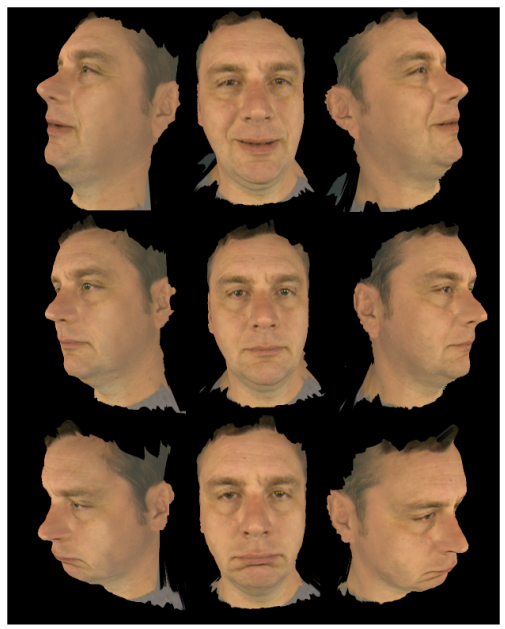

Figure 9: 3D Reconstructions of facial expressions

\section{Conclusion}

In this paper we have introduced a novel system for simultaneous video-rate capture of dense face shape and colour. Infra-red structured light illumination together with stereo is used to measure face shape. This work overcomes limitations of previous video-rate face measurement systems which use visible structured light pattern projection and markers. A novel displacement image representation based on an ellipsoidal mapping has been introduced for efficient integration and temporal registration of face shape. This provides a unified representation of dynamic face shape and colour as a video sequence of aligned displacement and colour images. Evaluation on the system on a database of 51 people performing facial expressions and speech demonstrates robust capture and representation of facial dynamics. Results show that the capture system achieves reconstruction of face shape with a visual quality comparable to the captured video. Future research will address the spatio- temporal characterisation of facial dynamics.

\section{References}

[1] P. Besl and N. McKay. A method for registration of 3-d shapes. IEEE Trans. Pattern Analysis and Machine Intelligence, 14(2):239-255, 1992.

[2] V. Blanz and T. Vetter. A Morphable Model for the Synthesis of 3D Faces. In Proc. ACM SIGGRAPH, pages 187-194, 1999.

[3] P. Fua. Regularized bundle-adjustment to model heads from image sequences without calibration data. International Journal of Computer Vision, 38(2):153-171, 2000.

[4] P. Fua and Y. Leclerc. Object-centred surface reconstruction: Combining multi-image stereo and shading. International Journal of Computer Vision, 16(1):35—56, 1995.

[5] A. Hilton, A.J. Stoddart, J. Illingworth, and T. Windeatt. Implicit surface based geometric fusion. International Journal of Computer Vision and Image Understanding, Special Issue on CAD Based Vision, 69(3):273-291, March 1998.

[6] G.A. Kalberer and L. Van Gool. Realistic face animation for speech. Journal of Visualization and Computer Animation.

[7] T. Kanade. Virtualized reality: putting reality into virtual reality. In 2nd International Workshop on Object Representation for Computer Vision ECCV, 1996.

[8] W.-S. Lee and N. Magnenat-Thalmann. Head Modeling from Pictures and Morphing in 3D with Image Metamorphosis Based on Triangulation. In Modelling and Motion Capture Techniques for Virtual Environments - Magnenat-Thalmann, $N$. and Thalmann,D. (Eds.), pages 254-268. Lecture Notes in Artificial Intelligence 1537, Springer Verlag, 1998.

[9] Z. Li, B. Curless, and S.M. Seitz. Spacetime stereo: Shape recovery of dynamic scenes. In Conference on Computer Vision and Pattern Recognition, 2003.

[10] P. Lindsey and A. Blake. Real-time tracking of surfaces with structured light. In British Machine Vision Conference, pages 619—628, 1994.

[11] S.K. Nayar, M. Watanabe, and M. Noguchi. Real-time focus range sensor. In International Conf. on Computer Vision, pages 9951001, 1995.

[12] M. Okutomi and T. Kanade. A locally adaptive window for signal matching. International Journal of Computer Vision, 7(2):143-162, 1992.

[13] F. Pighin, J. Hecker, D. Lischinski, R. Szeliski, and D.H. Salesin. Syntehsizing Realistic Facial Expressions From Photographs. In Proc. ACM SIGGRAPH, pages 75-84, 1998.

[14] M. Proesmans and L. VanGool. Active acquisition of 3d shape for moving objects. In International Conf. on Image Processing, pages 647-650, 1996.

[15] S. Rusinkiewicz, O. Hall-Holt, and M. Levoy. Real-time 3d model acquisition. In Proc. ACM SIGGRAPH, 2002.

[16] Y. Shan, Z. Liu, and Z. Zhang. Model-based bundle adjustment with application to face modeling. In International Conf. on Computer Vision, pages 644-651, 2001.

[17] M. Watanabe and S.K. Nayar. Telecentric optics for computer vision. In European Conference on Computer Vision, pages 439—451, 1995.

[18] Z. Zhang. A flexible new technique for camera calibration. IEEE Trans. Pattern Analysis and Machine Intelligence, 22(11):13301334, 2000. 